\title{
Autonomic Nervous System Dysfunction in Adolescents with Postural Orthostatic Tachycardia Syndrome and Chronic Fatigue Syndrome Is Characterized by Attenuated Vagal Baroreflex and Potentiated Sympathetic Vasomotion
}

\author{
JULIAN M. STEWART \\ Department of Pediatrics, The Center for Pediatric Hypotension, New York Medical College, Valhalla, \\ New York 10595, U.S.A.
}

\begin{tabular}{|c|c|}
\hline \multicolumn{2}{|c|}{ ABSTRACT } \\
\hline $\begin{array}{l}\text { The objective was to determine the nature of autonomic and } \\
\text { vasomotor changes in adolescent patients with orthostatic tachy- } \\
\text { cardia associated with the chronic fatigue syndrome (CFS) and } \\
\text { the postural orthostatic tachycardia syndrome (POTS). Continu- } \\
\text { ous electrocardiography and arterial tonometry was used to } \\
\text { investigate the heart rate and blood pressure responses before and } \\
3-5 \text { min after head-up tilt in } 22 \text { adolescents with POTS and } 14 \\
\text { adolescents with CFS, compared with control subjects compris- } \\
\text { ing } 10 \text { healthy adolescents and } 20 \text { patients with simple faint. } \\
\text { Heart rate and blood pressure variability, determined barorecep- } \\
\text { tor function using transfer function analysis, and measured car- } \\
\text { diac vagal and adrenergic autonomic responses were calculated } \\
\text { using timed breathing and the quantitative Valsalva maneuver. } \\
\text { Two of } 10 \text { healthy controls and } 14 \text { of } 20 \text { simple faint patients } \\
\text { experienced vasovagal syncope during head-up tilt. By design, } \\
\text { all CFS and POTS patients experienced orthostatic tachycardia, } \\
\text { often associated with hypotension. R-R interval and heart rate } \\
\text { variability were decreased in CFS and POTS patients compared } \\
\text { with control subjects and remained decreased with head-up tilt. } \\
\text { Low-frequency ( } 0.05-0.15 \text { Hz) blood pressure variability re- } \\
\text { flecting vasomotion was increased in CFS and POTS patients } \\
\text { compared with control subjects and increased further with } \\
\text { head-up tilt. This was associated with depressed baroreflex trans- } \\
\text { fer indicating baroreceptor attenuation through defective vagal }\end{array}$ & $\begin{array}{l}\text { efferent response. Only the sympathetic response remained. } \\
\text { Heart rate variability declined progressively from normal healthy } \\
\text { control subjects through syncope to POTS to CFS patients. } \\
\text { Timed breathing and Valsalva maneuver were most often normal } \\
\text { in CFS and POTS patients, although abnormalities in select } \\
\text { individuals were found. Heart rate and blood pressure regulation } \\
\text { in POTS and CFS patients are similar and indicate attenuated } \\
\text { efferent vagal baroreflex associated with increased vasomotor } \\
\text { tone. Loss of beat-to-beat heart rate control may contribute to a } \\
\text { destabilized blood pressure resulting in orthostatic intolerance. } \\
\text { The dysautonomia of orthostatic intolerance in POTS and in } \\
\text { chronic fatigue are similar. (Pediatr Res 48: 218-226, 2000) }\end{array}$ \\
\hline
\end{tabular}

Orthostatic intolerance is defined by the inability to remain upright without severe signs and symptoms such as hypotension, tachycardia, lightheadedness, pallor, fatigue, weakness, and nausea. Orthostatic intolerance appears in many guises including overt dysautonomia, vasovagal syncope, and orthostatic tachycardia (1). POTS is operationally defined by the

Received August 4, 1999; accepted January 26, 2000.

Correspondence: Julian M. Stewart, M.D., Ph.D., The Center for Pediatric Hypotension and Division of Pediatric Cardiology, Suite 618, Munger Pavilion, New York Medical College, Valhalla, NY 10595, U.S.A.

Supported in part by a grant from NIAID 1RO3-AI45954-01. presence of symptoms of orthostatic intolerance associated with an increase in sinus heart rate of $>30$ beats $/ \mathrm{min}$ or to a rate of $>120$ beats/min during the first $10 \mathrm{~min}$ of HUT table testing (2). Previous work, including our own, has suggested that adolescent patients with CFS often have findings of POTS during HUT (3-6). Conversely, patients with POTS often have fatigue as a prominent clinical feature $(2,7)$. Recent data have shown that adolescent patients with POTS share many symptoms with those having CFS, yet fail to satisfy the CDC criteria for that illness (8). Those criteria, which define the illness, are all symptom-related and include chronic debilitating fatigue 
lasting for $\geq 6$ mo associated with cognitive difficulties, pharyngitis, tender lymphadenopathy, muscle pain, joint pain, headache, sleep disturbance, and postexercise malaise unexplained by other illness. POTS children are usually less symptomatic, miss fewer school days, and generally recover within a matter of months (9). Prior studies showed a reduction in HRV consistent with either global autonomic dysfunction or vagal withdrawal $(5,6)$. However, lack of consistent autonomic testing and continuous BP recordings prohibit drawing conclusions concerning mechanism. To remedy this, the present studies were conducted to address the following questions:

1. Is there autonomic dysfunction in adolescent patients with orthostatic tachycardia?

2. Does this relate to patients' adaptation to orthostatic stress in the form of HUT?

3. What is the mechanism by which this alters the adaptation to orthostatic stress? Is there vagal withdrawal, sympathetic dysfunction, or some combination of both? Does this translate into altered baroreceptor function?

In the present investigation, data before and during HUT are presented for two groups of patients with orthostatic tachycardia: patients referred for the evaluation of CFS and fulfilling the CDC criteria for the syndrome, and patients with orthostatic intolerance and POTS. Data from these patients are compared with data from normal healthy control subjects and from patients with multiple episodes of simple faint using standard tests of autonomic function and measurements of HRV, BP variability, and cross-spectral analysis to assess baroreceptor function.

\section{METHODS}

Subjects. To accomplish these goals, 44 consecutive patients referred to pediatric cardiology for orthostatic intolerance who did not have a history of syncope were studied. Technically adequate tracings were obtained from 36 patients with orthostatic tachycardia, 14 of whom fit criteria for CFS (8). Data from only these 36 patients are presented. Patients had POTS on HUT. These were compared with 20 patients with multiple episodes $(>2)$ of simple faint who were symptom-free between episodes and with 10 normal control patients. Adequate tracings were obtained from all syncope patients and normal controls. Only children found during cardiac examination to be free from structural or arrhythmic heart disease were eligible to participate. Enrolled patients were free of all obvious systemic illnesses and were not taking neurally active or vasoactive medications. Informed consent was obtained, and all protocols were approved by the Committee for the Protection of Human Subjects of New York Medical College.

Patient classification. Patient classification of orthostatic tachycardia was in part defined a posteriori from HUT results. This is the most common orthostatic abnormality observed during HUT in adolescents with CFS $(5,9)$. Orthostatic intolerance was characterized by lightheadedness, nausea and vomiting, palpitations, fatigue, headache, blurred vision, abnormal sweating, and heat while upright. Significant hypotension dur- ing HUT was defined as $>30 \mathrm{~mm} \mathrm{Hg}$ fall in systolic BP. Significant relative bradycardia was defined as a heart rate $<75 \%$ of resting heart rate during HUT. Syncope was defined as significant hypotension with or without a significant relative bradycardia accompanied by loss of postural tone and loss of consciousness. Presyncope was defined as significant hypotension with or without a significant relative bradycardia but without loss of postural tone. POTS was diagnosed by symptoms of orthostatic intolerance during HUT associated with an increase in sinus heart rate of $>30$ beats $/ \mathrm{min}$ or to a rate of $>120$ beats/min during the first 10 min of tilt. Hypotension was not requisite. Criteria were selected for consistency with the adult literature $(2,7)$, but may not be entirely appropriate to adolescents. "Delayed orthostatic tachycardia syndrome" was defined during HUT if tachycardia with orthostatic intolerance occurred after $>10$ min of HUT.

POTS patients. The grouping of these 36 children was defined by their tilt table results. All complained of three or more symptoms of orthostatic intolerance for at least 3 mo. Twenty-two children with a diagnosis of POTS ranged in age from 12 to $17 \mathrm{y}$ (median, $15.2 \mathrm{y}$ ). Fourteen patients fulfilled the CDC criteria for CFS (8), as we have previously discussed (5, $6,9)$. There were 28 girls and eight boys. The group comprised 30 white children, four Asian children, and two black children. In what follows patients were stratified by CDC CFS criteria into two groups: CFS when criteria were present, and POTS when criteria were absent.

Healthy control patients. Ten volunteer patients, aged 13-17 y (median, $15.8 \mathrm{y}$ ), referred to pediatric cardiology for evaluation of a heart murmur determined to be innocent, were studied. Innocent murmur was defined by characteristic murmur(s), a normal ECG, and normal echocardiographic and Doppler findings (i.e. normal intracardiac and vascular anatomy, normal cardiac dimensions, and normal ventricular ejection). Patients with a history of syncope or orthostatic intolerance were specifically excluded. There were six girls and four boys. The group comprised seven white children, two Asian children, and one black child.

Syncope patients. Twenty patients, aged $12-18$ y (median, $16 \mathrm{y}$ ), referred to pediatric cardiology for evaluation of multiple syncope episodes, were studied. Patients were referred by area pediatricians for autonomic and tilt table evaluation. No cardiac, endocrine or vascular cause for episodes were found on initial evaluation and from historical data. We studied 13 girls and seven boys. The group comprised 14 white children, four Asian children, and two black children.

Patient assessment. All subjects were asked to fill out a questionnaire concerning symptoms of orthostatic intolerance, which incorporated items in the CDC definition of CFS, a review of systems, and scales to quantify the impact of fatigue and CFS on the subject's cognitive, physical, and social functioning. Pertinent laboratory tests included complete blood count with leukocyte differential; erythrocyte sedimentation rate; serum levels of alanine aminotransferase, total protein, albumin, globulin, alkaline phosphatase, calcium, phosphorus, glucose, blood urea nitrogen, electrolytes, and creatinine; determination of thyroid-stimulating hormone; and urinalysis, which were normal in every instance. Our orthostatic intoler- 
ance patients were also tested for Lyme disease with Lyme ELISA and Western blot because Lyme disease is endemic in this area. Patients with active Lyme disease were specifically excluded. A routine cardiovascular physical examination was performed and was supplemented by electrocardiographic and echocardiographic assessments to rule out heart disease.

Clinical autonomic nervous system assessment. All testing was performed in the laboratory during a single day. The methods have been previously described $(5,6,9)$. After overnight fast, tests began between 0900 and 1000. The ECG was monitored continuously and recorded to assess cardiac rhythm. Blood pressure was continuously monitored with an arterial tonometer (Collin Instruments, San Antonio, TX, U.S.A.) placed on the left radial artery, calibrated against oscillometric sphygmomanometer pressure. A continuous electrocardiographic recorder (Holter monitor, Marquette Systems 8000, Milwaukee, WI, U.S.A.) was also attached for rhythm verification. ECG and pressure data were interfaced to a personal computer through an A/D convertor (DataQ Inc., Milwaukee, WI, U.S.A.), and custom software was used to produce, display, and store R-R intervals and BP (mean, systolic, diastolic, and phasic tracings) on a continuous basis.

Heart rate changes with rhythmic breathing. Timed rhythmic breathing was performed in the supine position which yielded the most stable BP tracings. Equal inspirations and expirations at rates of 6,9 , and 12 breaths/min timed by a metronome were used, but only data at 6 breaths/min are reported for consistency with the adult literature (10). To quantify the relationship, the difference between maximum and minimum heart rate for eight consecutive beats was averaged. Heart rate difference data were also expressed in normalized form by dividing the difference by the resting heart rate.

Quantitative Valsalva maneuver. Subjects were asked to perform the Valsalva maneuver by blowing with an open glottis into a mouthpiece connected to the mercury column of a sphygmomanometer at $40-50 \mathrm{~mm} \mathrm{Hg}$ pressure. There was a built-in air leak to ensure an open glottis. After $15 \mathrm{~s}$, pressure was released, taking care to prevent deep breathing after release. The usual response during elevation of intrathoracic pressure was a transient increase in BP, followed by a fall in systolic pressure and pulse pressure as venous return decreases (early phase II), and compensatory vasoconstriction and tachycardia increasing the BP if autonomic reflexes are intact (late phase II). Late phase II therefore serves as a qualitative means to assess whether the vasoconstrictive effector arm of the baroreflex is intact. During release there is a transient fall in BP followed by pressure overshoot (phase IV) provided adrenergic vasoconstriction has occurred, and baroreceptor-mediated bradycardia if cardiac vagal reflexes are intact (11). Before the maneuver, BP was recorded and averaged for 20 beats, and mean resting heart rate was similarly calculated. The difference between longest and shortest R-R interval at rest was calculated. The tachycardia ratio (ratio of the shortest R-R interval during the test to the longest R-R interval before the test) was computed. The Valsalva ratio (ratio of the longest R-R interval after the maneuver divided by the shortest R-R interval during the test) was also calculated. In sympathetic constrictor failure, there is no stabilization of the BP during phase II of the maneuver and absence of overshoot with failure of bradycardic response during phase IV. In selective cardiac vagal failure, the overshoot may be present, but the corresponding bradycardia is lacking (11).

HUT table testing. An electrically driven tilt table (Cardiosystems 600, Dallas, TX, U.S.A.) with a foot board for weight bearing was used. Baseline recordings of $\mathrm{BP}$ and heart rate in the supine position were obtained near the end of a 30-min resting period. Patients were tilted to 70 degrees for a maximum duration of $30 \mathrm{~min}$ or until syncope, presyncope, or intolerable symptoms with or without significant hypotension (>30 mm Hg mean BP fall) occurred. Patients with a positive test were returned immediately to the supine position, and the test was terminated (12). No pharmacologic potentiation was used.

HRV and BP variability. At least 500 beats were acquired during baseline supine continuous recordings of heart rate and BP after a 30-min- resting period in all subjects. Beats were again acquired for 3-5 min after a 1- to 2-min BP stabilization period after HUT. Data were collected using a custom software package that enabled collection of a digital sequence containing R-R interval and systolic, diastolic, and mean BP for each heart beat. Separate analog BP and ECG data were digitized at $250 \mathrm{~Hz}$ and saved using custom software and an A/D convertor (DataQ). Continuous R-R interval and BP beat sequences were analyzed for ectopy, which was corrected by removing the ectopic beat and interpolating with a cubic spline for five beats in either direction. This was rarely necessary. Tachyarrhythmias or couplets were never observed. Beat epochs were linear detrended, and approximate wide-sense stationarity was obtained throughout tracings. Approximate wide-sense stationarity was verified by calculating autocorrelations and spectra of $\mathrm{R}-\mathrm{R}$ intervals in consecutive 200-beat subsets and comparing them with 500-beat records. No consistent trending was found. Time domain indices of HRV were calculated. These included the mean R-R interval, the SD of the R-R interval and the root mean square of successive RR differences. Time domain indices of systolic BP variability included the mean systolic BP and the SD of the systolic BP. An autoregressive model was used to calculate the R-R interval spectrum, BP power spectrum, and cross spectrum (13-15). In brief, R-R intervals and systolic, diastolic, and mean BP were acquired as a sequence of discrete point events. The sequences were transformed into an equivalent impulse train in which pulses were arranged at equal intervals (equal to the mean R-R interval) with impulse heights equal to the R-R intervals or BP as appropriate. Autoregression was performed, digital power spectra were calculated using the extended Yule-Walker equations, and the final order of the model was chosen to minimize Akaike's final prediction error (16). This yields the interval spectrum, which is converted to the spectrum of counts by dividing by the mean R-R interval of the sequence. The spectral power within a given band was computed by taking the power in the actual frequency band. Spectral power was partitioned into VLF $(0.01-0.04 \mathrm{~Hz}), \mathrm{LF}$ $(0.04-0.15 \mathrm{~Hz})$, and HF $(0.15-0.40 \mathrm{~Hz})$ power bands. The total power was also calculated. For purposes of the present analysis, only LF and HF bands were focused on for R-R interval variability, but VLF bands for BP are also presented. 
The LF systolic BP power band represents periodic oscillations, which may reflect intrinsic vasomotion. The LF R-R interval power band reflects smaller contributions directly from the sympathetic nervous system $(13,14)$, plus larger vagal efferent baroreceptor-mediated changes originating in the pressure trace (vasomotion), and therefore contains both sympathetic and parasympathetic components altering heart rate. The HF band represents the effects of parasympathetic modulation on heart rate and BP and also mechanical effects of respiration on cardiac filling and BP. The LF/HF ratio was calculated to represent overall sympathovagal balance (14). The normalized cross-spectrum between R-R interval and systolic BP was used to calculate the magnitude and phase of the transfer function between systolic BP and R-R interval as an index of baroreceptor gain when coherence exceeded $0.5(15,17)$. The square root of the ratio of LF R-R interval power to LF systolic BP power (18) was also calculated as a second estimate of LF baroreceptor gain and as an internal consistency check.

Protocol. Tests were done in the following order: $30 \mathrm{~min}$ of quiet supine resting, baseline heart rate and BP variability assessments, rhythmic breathing, Valsalva maneuver, a second baseline variability collection to rule out time dependence, and lastly, HUT with variability data collection. For purposes of comparison, R-R interval data, BP data, and baroreflex data were collected and computed during supine rest before the patients were tilted and during HUT.

Statistics. Data were compared by two-way ANOVA for repeated measures. When significant interactions were demonstrated, the ratio of $F$ values was converted to a $t$ distribution using Scheffe's test, and probabilities were thereafter determined. A Bonferroni correction was used to correct for small samples. All results are reported as mean \pm SEM. Statistically significant differences are reported for $p<0.05$.

\section{RESULTS}

Principal results are reported in Tables 1 and 2. Representative graphic depictions are found in Figures 1-3. All CFS and POTS patients had orthostatic tachycardia during HUT testing by study design. Of the healthy control patients, two of $10 \mathrm{had}$ typical vasovagal episodes (mixed vasodepressor-cardioinhibitory) with abrupt onset of bradycardia and hypotension $10 \mathrm{~min}$ and $15 \mathrm{~min}$ after HUT. Of the syncope patients, 14 had vasovagal episodes and six had normal tilt tests. A fall in BP always preceded bradycardia. No healthy or syncope control patients had orthostatic tachycardia. Early orthostatic tachycardia during HUT occurred in nine of 14 patients with CFS (six of nine, hypotensive), and delayed orthostatic tachycardia in five of 14 (three of five, hypotensive). Early POTS occurred in 13 of 22 POTS patients. Of these, eight of 13 were hypotensive. Hypotension was delayed in three of eight patients. Delayed POTS occurred in nine of 22; eight of nine were hypotensive.

$\boldsymbol{H} \boldsymbol{R} \boldsymbol{V}$. Resting data before HUT (Table 1) are similar to data previously reported for CFS. Indices of HRV and R-R interval are decreased in CFS patients compared with healthy control subjects and syncopal patients. Indices of HRV were related to clinical group and were highest in normal control subjects,
Table 1. $R-R$ interval variability during supine rest and during HUT

\begin{tabular}{|c|c|c|}
\hline Variable & During supine rest & During HUT \\
\hline \multicolumn{3}{|l|}{$\mathrm{LFP}\left(\mathrm{ms}^{2} / \mathrm{Hz}\right)$} \\
\hline CFS & $352 \pm 76 \dagger$ & $279 \pm 41 \dagger$ \\
\hline POTS & $543 \pm 145 \dagger$ & $387 \pm 60 \dagger$ \\
\hline Syncope & $866 \pm 123$ & $496 \pm 99^{*}$ \\
\hline Healthy & $1054 \pm 108$ & $602 \pm 49^{*}$ \\
\hline \multicolumn{3}{|c|}{$\mathrm{HFP}\left(\mathrm{ms}^{2} / \mathrm{Hz}\right)$} \\
\hline CFS & $540 \pm 113 \dagger$ & $124 \pm 29 * \dagger$ \\
\hline POTS & $778 \pm 196 \dagger$ & $178 \pm 46^{* \dagger}$ \\
\hline Syncope & $2168 \pm 461 \dagger$ & $293 \pm 77^{*}$ \\
\hline Healthy & $3123 \pm 301$ & $312 \pm 43^{*}$ \\
\hline \multicolumn{3}{|l|}{$\mathrm{TP}\left(\mathrm{ms}^{2} / \mathrm{Hz}\right)$} \\
\hline CFS & $1148 \pm 179 \dagger$ & $643 \pm 82 * \dagger$ \\
\hline POTS & $1823 \pm 449 \dagger$ & $792 \pm 112 * \dagger$ \\
\hline Syncope & $3573 \pm 540 \dagger$ & $1153 \pm 192 * \dagger$ \\
\hline Healthy & $4891 \pm 602$ & $2188 \pm 137^{*}$ \\
\hline \multicolumn{3}{|c|}{ Mean R-R (ms) } \\
\hline CFS & $793 \pm 27 \dagger$ & $593 \pm 15^{* \dagger}$ \\
\hline POTS & $793 \pm 28 \dagger$ & $633 \pm 19 * \dagger$ \\
\hline Syncope & $911 \pm 41$ & $706 \pm 27^{*}$ \\
\hline Healthy & $899 \pm 33$ & $798 \pm 14^{*}$ \\
\hline \multicolumn{3}{|l|}{$\mathrm{SD}(\mathrm{ms})$} \\
\hline CFS & $50 \pm 4 \dagger$ & $44 \pm 4^{* \dagger}$ \\
\hline POTS & $59 \pm 6 \dagger$ & $44 \pm 4^{* \dagger}$ \\
\hline Syncope & $85 \pm 6 \dagger$ & $53 \pm 4 * \dagger$ \\
\hline Healthy & $122 \pm 19$ & $78 \pm 9^{*}$ \\
\hline \multicolumn{3}{|l|}{ RMSSD (ms) } \\
\hline CFS & $45 \pm 5 \dagger$ & $20 \pm 2 * \dagger$ \\
\hline POTS & $51 \pm 6 \dagger$ & $23 \pm 3^{* \dagger}$ \\
\hline Syncope & $96 \pm 10$ & $32 \pm 4^{*}$ \\
\hline Healthy & $116 \pm 14$ & $39 \pm 5^{*}$ \\
\hline \multicolumn{3}{|c|}{ pNN50 (percent) } \\
\hline CFS & $24 \pm 4 \dagger$ & $2 \pm 1 * \dagger$ \\
\hline POTS & $25 \pm 5 \dagger$ & $6 \pm 2 * \dagger$ \\
\hline Syncope & $50 \pm 4$ & $10 \pm 3 *$ \\
\hline Healthy & $67 \pm 6$ & $17 \pm 3^{*}$ \\
\hline \multicolumn{3}{|l|}{$\mathrm{LF} / \mathrm{HF}$} \\
\hline CFS & $0.81 \pm 0.15 \dagger$ & $3.19 \pm 0.53 * \dagger$ \\
\hline POTS & $0.94 \pm 0.16 \dagger$ & $3.14 \pm 0.45^{* \dagger} \dagger$ \\
\hline Syncope & $0.70 \pm 0.11$ & $2.59 \pm 0.32 *$ \\
\hline Healthy & $0.54 \pm 0.11$ & $2.33 \pm 0.47^{*}$ \\
\hline \multicolumn{3}{|l|}{ LFNU } \\
\hline CFS & $0.29 \pm 0.02$ & $0.45 \pm 0.04^{*}$ \\
\hline POTS & $0.30 \pm 0.02$ & $0.47 \pm 0.03 *$ \\
\hline Syncope & $0.28 \pm 0.04$ & $0.42 \pm 0.02 *$ \\
\hline Healthy & $0.38 \pm 0.06$ & $0.40 \pm 0.02$ \\
\hline \multicolumn{3}{|l|}{ HFNU } \\
\hline CFS & $0.45 \pm 0.05 \dagger$ & $0.18 \pm 0.02 * \dagger$ \\
\hline POTS & $0.43 \pm 0.03 \uparrow$ & $0.21 \pm 0.02 *$ \\
\hline Syncope & $0.54 \pm 0.04$ & $0.22 \pm 0.03 *$ \\
\hline Healthy & $0.66 \pm 0.03$ & $0.29 \pm 0.02 *$ \\
\hline
\end{tabular}

LFP, LF power; HFP, HF power; TP, total power; SD, standard deviation of $\mathrm{R}-\mathrm{R}$ intervals; RMSSD, root mean square successive difference of R-R intervals; pNN50, percent normal R-R interval differences $>50 \mathrm{~ms}$; $\mathrm{LF} / \mathrm{HF}$, ratio of LF to HF power; LFNU, normalized LF power; HFNU, normalized HF power.

$* p<0.05$ compared with before tilt; $\dagger p<0.05$ compared with healthy control.

somewhat lower in syncope patients, significantly decreased compared with both control groups in POTS patients, and further decreased in CFS. This relation was maintained during HUT. The LF/HF ratio was significantly increased in CFS and POTS groups compared with control subjects, indicating enhanced sympathovagal balance. All indices decreased significantly compared with baseline with HUT. 
Table 2. Timed breathing and quantitative Valsalva maneuver

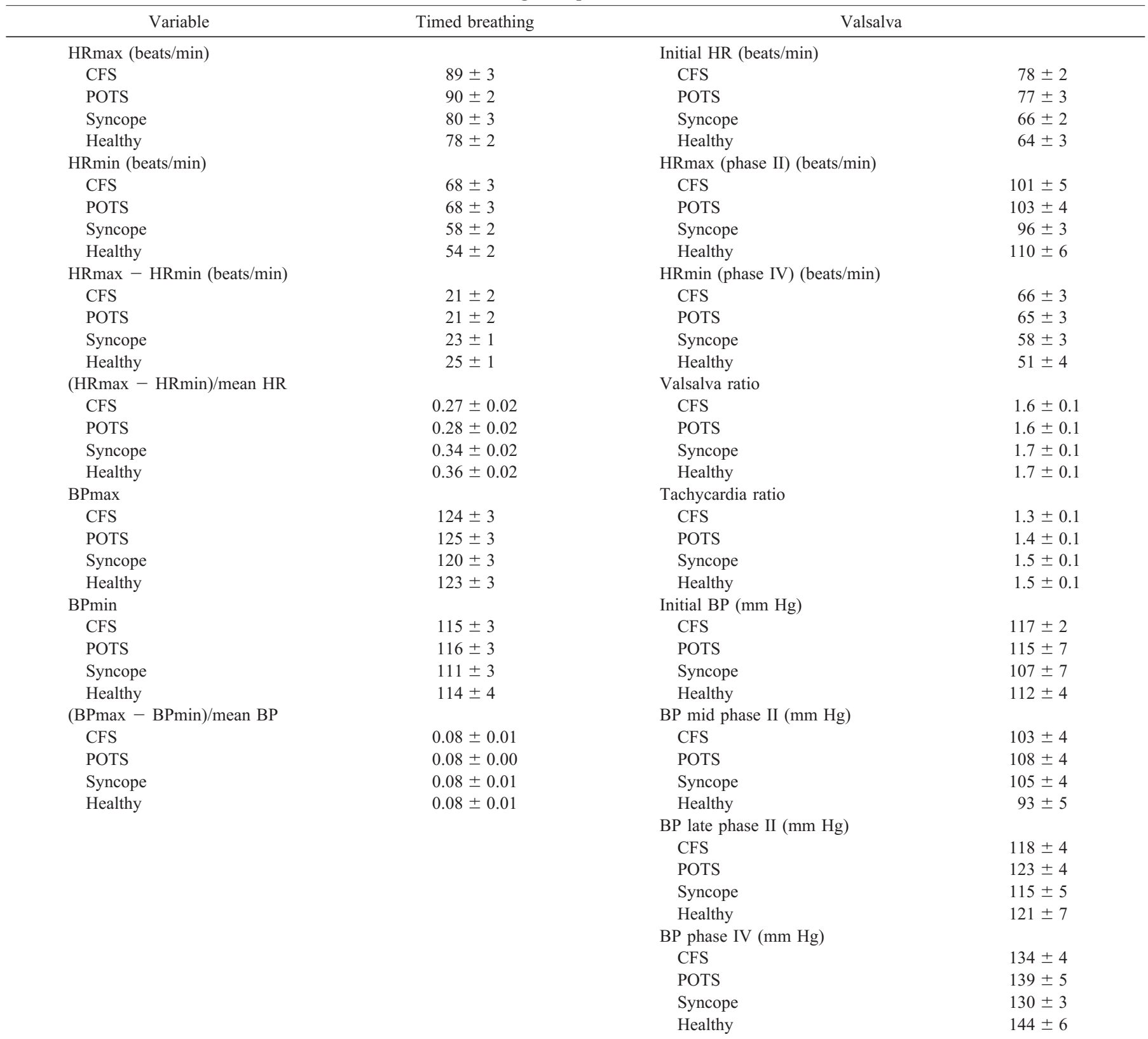

HRmax, maximum heart rate during timed breathing at 6 breaths/min; HRmin, minimum heart rate during timed breathing; BPmax, maximum BP during timed breathing; BPmin, minimum BP during timed breathing; initial HR, initial heart rate before start of Valsalva maneuver; HRmax (phase II), maximum heart rate during phase II of Valsalva maneuver; HRmin (phase IV), minimum heart rate during phase IV of the Valsalva maneuver; BP abbreviations are self explanatory.

$\dagger p<0.05$ compared with healthy control.

$\mathrm{R}-\mathrm{R}$ interval and BP tachograms and their respective spectra are presented in Figures 1 and 2, which compare a representative POTS patient with a representative healthy control patient. CFS and syncopal representations are similar. There is a decrease in overall variability in R-R interval tachogram in Figure 1 for POTS, whereas Figure 2 shows a greatly decreased HF peak in HRV for POTS and some diminution of the LF peak for POTS.

BP variability. Baseline $B P$ variability was similar in control groups compared with CFS and POTS patients, although LF power (representing intrinsic vasomotion) was somewhat increased in the CFS and POTS groups (Table 3). These differences can also be observed in Figures 1 and 2, which contrast the BP variation in representative control and POTS patients. With HUT, these differences were magnified, with more marked increases in LF spectral power (vasomotion) in CFS and POTS. There was also a large component of overall variability in the VLF and ultra LF bands representing slow changes, possibly of thermoregulatory or neuroendocrine origin. Although these findings are interesting, they are beyond the scope of the present investigation.

Baroreceptor data. There were significant decreases in baroreceptor transfer function magnitude (i.e. baroreceptor gain) in CFS and POTS, which was most evident in the HF range (Table 4). The changes are depicted for representative control and POTS patients in Figure 3, which shows high gain 

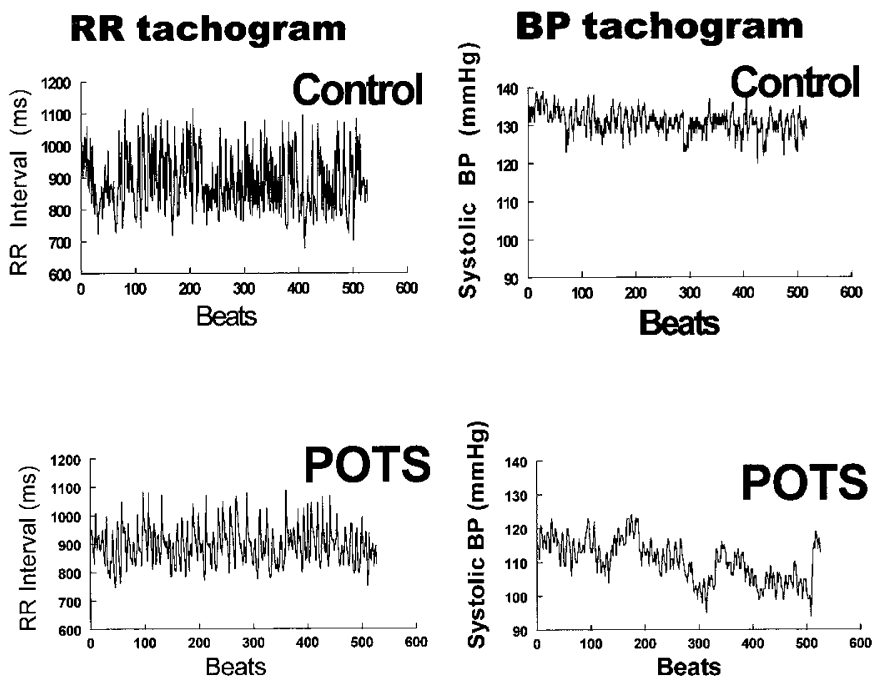

Figure 1. Baseline R-R interval tachograms (Left) and systolic BP tachograms (Right) are shown for representative patients. Top, healthy control subject (control) who was not syncopal during HUT table testing. Bottom, patient with POTS. Results obtained from CFS patients are similar to POTS results. HRV appears to be decreased in POTS compared with control, whereas $\mathrm{BP}$ variation appears to be increased.

\section{RR spectra}
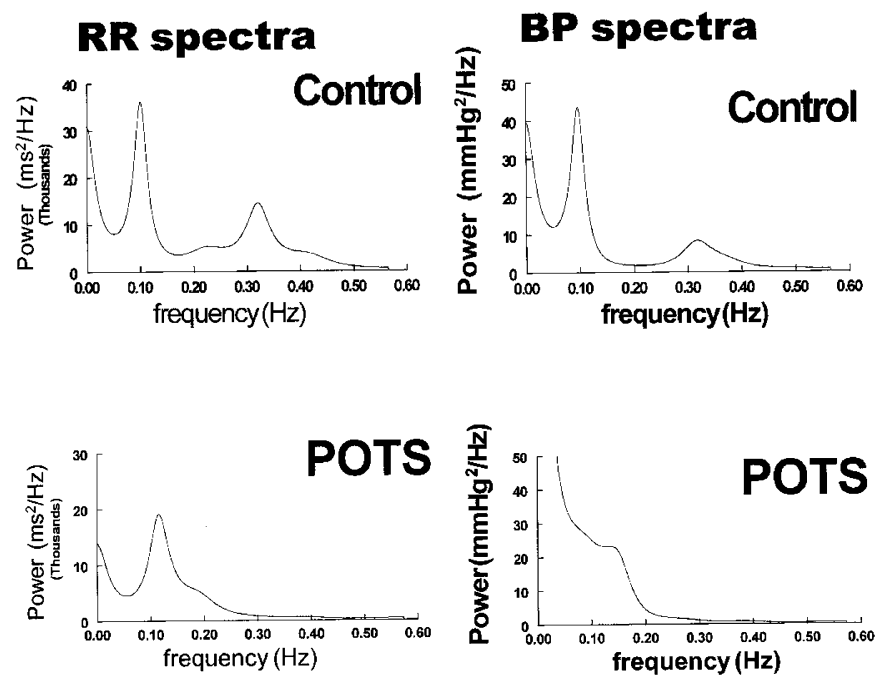

Figure 2. Frequency domain data (spectra) corresponding to R-R interval and BP tachograms of Figure 1. Left, R-R interval spectra. Right, BP spectra. Top, healthy control subject (control). Bottom, patient with POTS. HF spectral R-R interval power is almost completely attenuated for POTS and attenuated at LF. $\mathrm{BP}$ variability is increased at LF domain.

and coherence throughout the spectral region of interest (relatively flat transfer factor) for the normal control patient but greatly diminished HF gain and coherence for the representative POTS patient. The phase plot for the POTS patient shows an abrupt change in phase at the HF peak, at which phase shifts from approximately 180 degrees to 0 degrees and then back to 180 degrees again - unstable indeed.

Autonomic data. Table 2 shows averaged results for timed breathing and Valsalva maneuver in the groups. There is little significant difference noted, although maximum and minimum heart rates tended to be higher in CFS and POTS patients compared with controls. The averaged data obscure individual patient results, however. In selected cases, CFS and POTS
Coherence Transfer Phase Transfer Magnitude
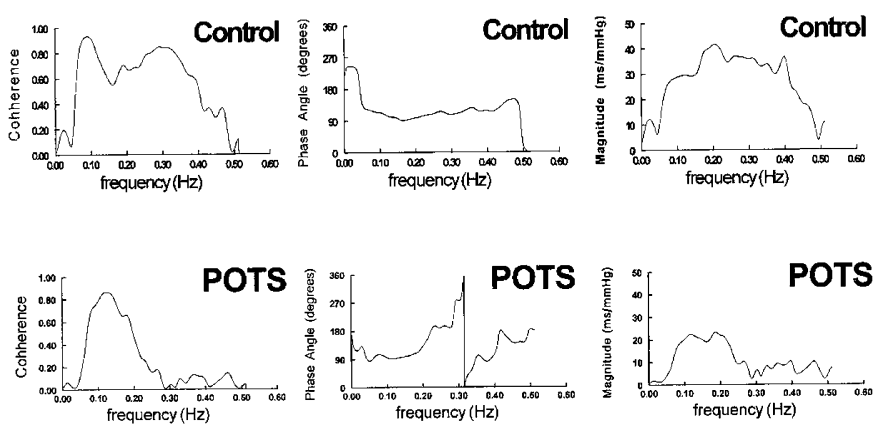

Figure 3. Baroreceptor transfer function data correspond to R-R interval and BP tachograms of Figures 1 and 2. Left, coherence function. Middle, transfer function phase as a function of frequency. Right, transfer function magnitude as a function of frequency. Top, control data. Bottom, POTS data. Transfer function magnitude, corresponding to baroreceptor gain, is blunted in POTS compared with control. Only LF transfer occurs. There is an abrupt oscillation in phase near the respiratory peak $(0.30 \mathrm{~Hz})$.

patients had greatly decreased HRV during breathing, depressed Valsalva ratio, and decreased late phase II of the Valsalva maneuver, often associated with little alteration in phase-IV heart rate.

\section{DISCUSSION}

The major findings of this study are the following:

1. Orthostatic tachycardia occurs in CFS and is qualitatively indistinguishable from orthostatic tachycardia associated with POTS.

2. Resting R-R interval and HRV are decreased in CFS and POTS compared with control group and remain decreased after HUT.

3. Resting BP is not different. LF BP spectral power and total $\mathrm{BP}$ variability is increased in CFS and POTS compared with control group and is further increased by HUT.

4. The vagal efferent (RR-systolic BP transfer) arm of the baroreflex response is attenuated in CFS and POTS compared with control group before and after HUT.

5. There is progressive decrease in HRV from normal healthy control group to syncope group to POTS to CFS. However, autonomic abnormalities are similar in POTS and CFS.

6. Traditional cardiac vagal (timed breathing and Valsalva) and adrenergic (Valsalva) autonomic tests are frequently unaffected in CFS and POTS, although individual abnormalities are sometimes found.

Orthostatic tachycardia in CFS is qualitatively and quantitatively indistinguishable from orthostatic tachycardia associated with POTS. On the simplest level, these data show a relation between the autonomic findings in POTS and findings in CFS. This suggests that adolescents with POTS have symptoms similar in many respects to children with CFS, and have patterns of HRV and BP variability similar to children with CFS. I and my colleagues have reported HRV data before (5, 6). Others have also reported a relation between POTS and CFS (19) and have demonstrated an association with neurally 
Table 3. Blood pressure variability during supine rest and during HUT

\begin{tabular}{|c|c|c|}
\hline Variable & During supine rest & During HUT \\
\hline \multicolumn{3}{|c|}{$\operatorname{VLFP}\left(\mathrm{mm} \mathrm{Hg}^{2} / \mathrm{Hz}\right)$} \\
\hline $\mathrm{CFS}$ & $3.0 \pm 0.8 \dagger$ & $7.0 \pm 1.0 * \dagger$ \\
\hline POTS & $4.9 \pm 2.9$ & $6.3 \pm 1.3$ \\
\hline Syncope & $2.6 \pm 0.3$ & $5.7 \pm 0.9 *$ \\
\hline Healthy & $1.6 \pm 0.2$ & $4.3 \pm 0.7^{*}$ \\
\hline \multicolumn{3}{|c|}{$\mathrm{LFP}\left(\mathrm{mm} \mathrm{Hg}^{2} / \mathrm{Hz}\right)$} \\
\hline CFS & $1.1 \pm 0.2$ & $5.9 \pm 1.6^{* \dagger}$ \\
\hline POTS & $3.3 \pm 2.1 \dagger$ & $7.1 \pm 1.7^{* \dagger}$ \\
\hline Syncope & $1.4 \pm 0.3$ & $4.9 \pm 0.8^{*}$ \\
\hline Healthy & $1.0 \pm 0.2$ & $3.6 \pm 0.9^{*}$ \\
\hline \multicolumn{3}{|c|}{$\mathrm{HFP}\left(\mathrm{mm} \mathrm{Hg} \mathrm{Hg}^{2} / \mathrm{Hz}\right)$} \\
\hline CFS & $1.0 \pm 0.2$ & $2.8 \pm 0.5^{*}$ \\
\hline POTS & $1.5 \pm 0.3$ & $3.7 \pm 0.7^{*}$ \\
\hline Syncope & $1.3 \pm 0.2$ & $2.6 \pm 0.3^{*}$ \\
\hline Healthy & $1.3 \pm 0.1$ & $2.7 \pm 0.2 *$ \\
\hline \multicolumn{3}{|c|}{$\mathrm{TP}(\mathrm{mm} \mathrm{Hg} / \mathrm{Hz})$} \\
\hline CFS & $7.1 \pm 1.1$ & $16.7 \pm 2.6^{*} \dagger$ \\
\hline POTS & $9.3 \pm 1.5 \dagger$ & $17.2 \pm 2.1^{*} \dagger$ \\
\hline Syncope & $5.2 \pm 0.9$ & $13.2 \pm 1.1^{*}$ \\
\hline Healthy & $5.9 \pm 0.8$ & $11.9 \pm 1.0^{*}$ \\
\hline \multicolumn{3}{|c|}{ Systolic BP (mm Hg) } \\
\hline CFS & $115 \pm 3$ & $104 \pm 3$ \\
\hline POTS & $119 \pm 4$ & $112 \pm 4$ \\
\hline Syncope & $113 \pm 3$ & $107 \pm 2$ \\
\hline Healthy & $115 \pm 6$ & $116 \pm 3$ \\
\hline \multicolumn{3}{|l|}{$\mathrm{SD}(\mathrm{mm} \mathrm{Hg})$} \\
\hline CFS & $4.6 \pm 0.2 \dagger$ & $8.1 \pm 0.6^{* \dagger}$ \\
\hline POTS & $4.7 \pm 0.3$ & $7.5 \pm 0.5^{* \dagger}$ \\
\hline Syncope & $4.0 \pm 0.2$ & $6.6 \pm 0.3^{*}$ \\
\hline Healthy & $4.1 \pm 0.2$ & $5.6 \pm 0.3^{*}$ \\
\hline \multicolumn{3}{|l|}{$\mathrm{LF} / \mathrm{HF}$} \\
\hline CFS & $1.1 \pm 0.2$ & $2.0 \pm 0.3^{*}$ \\
\hline POTS & $1.2 \pm 0.3$ & $2.0 \pm 0.3$ \\
\hline Syncope & $1.1 \pm 0.2$ & $1.9 \pm 0.2 *$ \\
\hline Healthy & $1.1 \pm 0.2$ & $2.0 \pm 0.3^{*}$ \\
\hline \multicolumn{3}{|l|}{ LFNU } \\
\hline CFS & $0.24 \pm 0.02$ & $0.34 \pm 0.04$ \\
\hline POTS & $0.22 \pm 0.02$ & $0.40 \pm 0.03 *$ \\
\hline Syncope & $0.23 \pm 0.02$ & $0.35 \pm 0.02 *$ \\
\hline Healthy & $0.22 \pm 0.02$ & $0.32 \pm 0.02 *$ \\
\hline \multicolumn{3}{|l|}{ HFNU } \\
\hline CFS & $0.25 \pm 0.03$ & $0.19 \pm 0.02$ \\
\hline POTS & $0.30 \pm 0.03$ & $0.24 \pm 0.02$ \\
\hline Syncope & $0.33 \pm 0.04$ & $0.23 \pm 0.02$ \\
\hline Healthy & $0.35 \pm 0.03$ & $0.25 \pm 0.01 *$ \\
\hline
\end{tabular}

VLFP, VLF power; LFP, LF power; HFP, HF power; TP, total power; SD, standard deviation of R-R intervals; $L F / H F$, ratio of LF to HF power; LFNU, normalized LF power; HFNU, normalized HF power.

$* p<0.05$ compared with before tilt; $\dagger p<0.05$ compared with healthy control.

mediated hypotension $(3,4)$, although results are variable $(20$, 21).

Resting HRV is decreased in CFS and POTS compared with control and remains decreased after HUT. I and my colleagues have reported this before $(5,6,9)$. The observation remains at variance with adult data concerning CFS (20). Decreased HRV has been described in severely ill adult POTS patients of Low et al. (22) and Novak et al. (23), who used elegant computer techniques to probe spectral properties. The current data show persistence of the LF peak in the HRV spectrum, albeit at decreased magnitude. This suggests atten-
Table 4. Baroreceptor data during supine rest and during HUT

\begin{tabular}{lcc}
\hline \multicolumn{1}{c}{ Variable } & During supine rest & During HUT \\
\hline Alpha $(\mathrm{ms} / \mathrm{mm} \mathrm{Hg})$ & & \\
CFS & $19 \pm 2 \dagger$ & $8 \pm 1^{* \dagger}$ \\
POTS & $23 \pm 3 \dagger$ & $8 \pm 1^{* \dagger}$ \\
Syncope & $32 \pm 3$ & $12 \pm 2$ \\
Healthy & $35 \pm 3$ & $16 \pm 2$ \\
LF Transfer Function Magnitude (gain) & & \\
CFS & $16 \pm 3 \dagger$ & $4 \pm 1^{*} \dagger$ \\
POTS & $19 \pm 3 \dagger$ & $7 \pm 1$ \\
Syncope & $26 \pm 3 \dagger$ & $8 \pm 1$ \\
Healthy & $38 \pm 2$ & $10 \pm 2$ \\
HF Transfer Function Magnitude (gain ms/mm Hg) & \\
CFS & $19 \pm 2 \dagger$ & $4 \pm 1^{*} \dagger$ \\
POTS & $22 \pm 4 \dagger$ & $5 \pm 1^{* \dagger}$ \\
Syncope & $32 \pm 4$ & $8 \pm 1$ \\
Healthy & $37 \pm 3$ & $9 \pm 1$ \\
\hline Alpha, ratio of average LF R-R interval power to LF systolic BP power. \\
$* p<0.05$ compared with before tilt; $\dagger p<0.05$ compared with healthy \\
control.
\end{tabular}

uation of the vagally mediated efferent baroreflex arm to the sinus node, although sympathetic stimulation of the heart may be preserved. Increased LF/HF ratio supports this idea, as does variability in BP and baroreceptor data discussed below. Separate support is contained in the work of Pagani et al. (24), who found a shift toward sympathetic dominance in sympathovagal balance in adults with CFS compared with healthy control subjects using autoregressive methodology similar to that used in the current study.

$L F B P$ spectral power and total BP variability is increased in CFS and POTS before tilt compared with control and is further increased after HUT. There is enhanced vasomotor tone as indicated by increased BP variability in CFS and POTS compared with control. The data suggest an intact sympathetic vascular efferent arm of the baroreflex. Intact Valsalva phase II supports the inference. A separate contribution to increased BP variability may arise from decreased HRV. It is known that heart rate changes influence diastolic BP (25); they may also exert an effect on systolic BP through changes in cardiac filling times and through the Treppe phenomenon. We believe that $\mathrm{R}-\mathrm{R}$ interval variations serve to prevent rapid oscillations in $\mathrm{BP}$, and decreased R-R interval variability destabilizes BP and increases BP variability. Enhanced sympathetic activity is consistent with results from other laboratories: Streeten (26) defined the disease state of hyperadrenergic orthostatic hypotension with modestly enhanced norepinephrine and epinephrine plasma levels. Jacob et al. (27) noted decreased blood volume associated with increased plasma catecholamines. Furlan et al. (28) demonstrated increased muscle sympathetic nerve activity, increased LF/HF, and increased LF BP spectral power in their patients with chronic orthostatic intolerance and orthostatic tachycardia. These patients also had decreased HRV in the upright position.

$R-R$ interval-systolic BP transfer magnitude of the baroreflex in CFS and POTS is attenuated compared with control. Figure 3 shows marked attenuation of the baroreceptor transfer magnitude representing baroreceptor gain at HF, and a decrease at LF in POTS and CFS compared with control. This is consistent with complete vagal withdrawal and is similar to 
results reported by Saul et al. (29). Those investigators used atropine to block the vagus while measuring heart rate-systolic BP transfer function properties and demonstrated complete loss of transfer magnitude at HF and attenuation at LF. The current data support the hypothesis that increased BP variability in POTS and CFS results from increased vasomotion compounded by the loss of compensatory changes in heart rate in POTS and CFS. The technique of baroreceptor assessment via transfer function analysis has been tested and found to be sound $(15,17)$

Progressive decrease in HRV from normal healthy control to syncope to POTS to CFS. Patients with syncope, POTS, and CFS have distinct modes of clinical presentation. Nevertheless, there is considerable overlap in complaints, with features common to all forms of orthostatic intolerance. The response to orthostatic stress is similar. During HUT, the current study and others have also observed features similar to vasovagal syncope: patients with typical symptomatic orthostatic tachycardia may terminate testing with a classic vasovagal faint. Although it may be that enhanced sympathetic stimulation and vagal withdrawal sets the stage for such a response, one proposal is that there is a more fundamental similarity in pathophysiology with abnormal blood volume control (30), abnormal autonomic response to alterations in central blood volume, and abnormal CNS integration of baroreceptor, volume, and chemoreflex signals $(31,32)$.

Timed breathing and Valsalva maneuver are not often abnormal in CFS and POTS. In selected cases, both timed breathing and the Valsalva maneuver may be abnormal: several patients had a loss of late phase II recovery of BP associated with blunted heart rate increase in phase II and blunted variation in heart rate during timed breathing. However, such patients proved to be the exception rather than the rule. For the most part, the Valsalva maneuver was normal with an excellent phase II recovery, indicating intact adrenergic vasoconstriction, and patients also had a normal Valsalva ratio and tachycardia ratio, and normal HRV with breathing. This conflicts with the assertion of vagal withdrawal and is at some variance with the literature $(19,33)$ but may implicate altered respiratory modulation of vagal tone.

Attaching precise physiologic meaning to HRV data has been difficult. Although changes in LF and HF spectra may relate to baroreceptor function and parasympathetic factors (respiratory sinus arrhythmia), a precise correspondence is difficult to achieve. This difficulty may be partly alleviated by including BP variability, which may more accurately reflect vasomotor activity and sympathetic autonomic tone.

Another problem is how to account for patient selection bias, which is invariably present. In a sense, such bias was controlled for by selecting patients on the basis of clinical syndrome and tilt table result. Nevertheless, patients seen in an academic setting may differ considerably from those seen in a regular clinical practice.

Respiratory frequency and volume have a great impact on spectral analysis results, and, similarly, $\mathrm{CO}_{2}$ levels can affect spectral analysis. These quantities were not explicitly measured in the study. However, differences were apparent between POTS patients and control subjects at rest when there was no difference in appreciated respiratory rate, nor were there any marked differences during early tilt, i.e. the period of observation.

The finding of vagal withdrawal by variability analysis is at some odds with normal quantitative Valsalva maneuver. Although it is true that the Valsalva maneuver, on the average, did not show statistically significant differences, in some cases individual CFS and POTS patients had clearly abnormal Valsalva ratios. These were insufficient for statistical significance in the small groups used and thus a $\beta$-error of a sort has occurred. Variability analysis may be a more sensitive way to detect baroreflex dysfunction.

A final difficulty relates to defining tachycardia during HUT in adolescents. Adult standards indicate a normal upper limit of 30 beats/min within the first 10 min of tilt. It is likely that the 95\% confidence level for the adolescents in this study falls between 35 and 40 beats/min. However, there is insufficient normative to make this statement with assurance. On the other hand, the large majority of these patients would make the higher cut, and all patients were highly symptomatic, fulfilling criteria for orthostatic intolerance.

In summary, autonomic function and clinical features are similar in CFS and POTS. The data suggest vagal withdrawal with intact vasoactive baroreflexes and sympathoexcitation, which may contribute to vasomotor instability and orthostatic intolerance in these patients. A similar hypothesis concerning autonomic dysfunction based on other data in adults has been recently proposed by Pagani et al. (34).

Acknowledgment. The author thanks Amy Weldon for technical assistance and Dr. Michael H. Gewitz for his continued support in this endeavor.

\section{REFERENCES}

1. Grubb BP 1998 Neurocardiogenic syncope, and dysautonomic (orthostatic) syncope. In: Grubb BP, Olshansky B (eds) Syncope: Mechanisms and Management. Futura, Armonk, NY, pp 73-126

2. Schondorf R, Low PA 1993 Idiopathic postural orthostatic tachycardia syndrome: an attenuated form of acute pandysautonomia? Neurology 43:132-137

3. Rowe PC, Bou-Holaigh I, Kan JS, Calkins H 1995 Is neurally mediated hypotension an unrecognized cause of chronic fatigue? Lancet 345:623-624

4. Bou-Holaigh I, Rowe PC, Kan JS, Calkins H 1995 The relationship between neurally mediated hypotension and the chronic fatigue syndrome. JAMA 274:961-967

5. Stewart JM, Gewitz MH, Weldon A, Arlievsky N, Li K, Munoz J 1999 Orthostatic intolerance in adolescent chronic fatigue syndrome. Pediatrics 103:116-121

6. Stewart JM, Weldon A, Arlievsky N, Li. K, Munoz J 1998 Neurally mediated hypotension and autonomic dysfunction measured by heart rate variability during head-up tilt testing in children with the chronic fatigue syndrome. Clin Auton Res $8: 221-230$

7. Grubb BP, Kosinski DJ, Boehm K, Kip K 1997 Postural orthostatic tachycardia syndrome: a neurocardiogenic variant identified during head-up tilt table testing. Pacing Clin Electrophysiol 20:2205-2212

8. Fukuda K, Straus SE, Hickie I, Sharpe MC, Dobbins JG, Komaroff A 1994 The chronic fatigue syndrome: a comprehensive approach to its definition and study. Ann Intern Med 121:953-959

9. Stewart JM, Weldon A, Munoz J 1999 Patterns of orthostatic intolerance. The Orthostatic Tachycardia Syndrome and adolescent chronic fatigue. J Pediatr 135:218-225

10. Assessment Clinical Autonomic Testing Report of the Therapeutics and Technology Assessment Subcommittee of the American Academy of Neurology. 1991 Neurology 46:873-880

11. Smith ML, Beightol LA, Fritsch-Yelle JM, Ellenbogen KA, Porter TR, Eckberg DL 1996 Valsalva's maneuver revisited: a quantitative method yielding insights into human autonomic control. Am J Physiol 271:H1240-H1249

12. Benditt DG, Ferguson DW, Grubb BP, Kapoor WN, Kugler J, Lerman BB Maloney JD, Raviele A, Ross B, Sutton R, Wolk MJ, Wood DL 1996 Tilt table 
testing for assessing syncope: ACC expert consensus document. J Am Coll Cardiol 28:263-275

13. Montano N, Ruscone TG, Porta A, Lombardi F, Pagani M, Malliani A 1994 Power spectrum analysis of heart rate variability to assess the changes in sympathovagal balance during graded orthostatic tilt. Circulation 90:1826-1831

14. Pagani M, Lombardi F, Guzzeti S, Rimoldi O, Furlan R, Pizzinelli P, Sandrone G, Malfatto G, Dell'Orto S, Piccaluga E, Turiel M, Baselli G, Cerutti S, Malliani A 1986 Power spectral analysis of heart rate and arterial pressure variabilities as a marker of sympatho-vagal interaction in man and conscious dog. Circ Res 59:178-193

15. Robbe HWJ, Mulder LJM, Ruddel H, Langewitz WA, Veldman JBP, Mulder G 1987 Assessment of baroreceptor reflex sensitivity by means of spectral analysis. Hypertension 10:538-543

16. Kay SM, Marple SL 1981 Spectrum analysis - a modern perspective. Proc IEEE 69:1380-1419

17. Saul JP, Berger RD, Albrecht P, Stein SP, Chen MH, Cohen RJ 1991 Transfer function analysis of the circulation: unique insights into cardiovascular regulation. Am J Physiol 261:H1231-H1245

18. Pagani M, Somers V, Furlan R, Dell'Orto S, Conway J, Baselli G, Cerutti S, Sleight $\mathrm{P}$, Malliani A 1988 Changes in autonomic regulation induced by physical training in mild hypertension. Hypertension 12:600-610

19. DeLorenzo F, Hargreaves J, Kakkar VV 1996 Possible relationship between chronic fatigue and postural tachycardia syndromes. Clin Auton Res 6:263-264

20. Freeman R, Komaroff AL 1997 Does the chronic fatigue syndrome involve the autonomic nervous system? Am J Med 102:357-364

21. Sneddon JF, Counihan PJ, Bashir Y, Haywood GA, Ward DE, Camm AJ 1993 Assessment of autonomic function in patients with neurally mediated syncope: augmented cardiopulmonary baroreceptor responses to graded orthostatic stress. J Am Coll Cardiol 21:1193-1198

22. Low PA, Novak V, Novak P, Sandroni P 1997 Postural tachycardia syndrome. In: Low PA (ed) Clinical Autonomic Disorders. Lippincott-Raven, Philadelphia, pp 681-699
23. Novak V. Opfer-Gehrking TL, Low PA 1996 Postural tachycardia syndrome: timefrequency mapping. J Auton Nerv Syst 612:313-320

24. Pagani M, Lucini D, Mela GS, Langewitz W, Malliani A 1994 Sympathetic overactivity in subjects complaining of unexplained fatigue. Clin Sci 87:655-661

25. Barbieri R, Bianchi A, Triedman JK, Mainardi LT, Cerutti S, Saul JP. 1997 Mode dependency of multivariate autoregressive spectral analysis: quantifying cardiovascular control using bivariate and trivariate models. IEEE Eng Med Biol Mag $16: 74-85$

26. Streeten DP 1990 Pathogenesis of hyperadrenergic orthostatic hypotension. J Clin Invest 86:1582-1588

27. Jacob G, Biaggioni I, Mosqueda-Garcia R, Robertson RM, Robertson D 1998 Relation of blood volume and blood pressure in orthostatic intolerance. Am J Med Sci 315:95-100

28. Furlan G, Jacob G, Snell M, Robertson D, Porta A, Harris P, Mosqueda-Garcia R 1998 Chronic orthostatic intolerance: a disorder with discordant cardiac and vascular sympathetic control. Circulation 98:2154-2159

29. Saul JP, Berger RD, Triedman JK 1991 Transfer function analysis: insights into heart rate and blood pressure control. In: Hagen H, Koepchen HP (eds) Rhythms in Physiological Systems. Springer Verlag, Heidelberg, pp 103-114

30. Jacob G, Robertson D, Mosqueda-Garcia R, Ertl AC, Robertson RM, Biaggioni 1997 Hypovolemia in syncope and orthostatic intolerance: role of the renin angiotensin system. Am J Med 103:128-133

31. Low PA, Novak V, Spies JM, Novak P, Petty GW 1999 Cerebrovascular regulation in the postural orthostatic tachycardia syndrome (POTS). Am J Med Sci 317:124-133

32. Novak V, Spies JM, Novak P, McPhee BR, Rummans TA, Low PA 1998 Hypocapnia and cerebral hypoperfusion in orthostatic intolerance. Stroke 29:1876-1881

33. Sisto SA, Tapp W, Drastal S, Bergen M, DeMasi I, Cordero D, Natelson B 1995 Vagal tone is reduced during paced breathing in patients with the chronic fatigue syndrome. Clin Auton Res 5:139-43

34. Pagani M, Lucini D 1999 Chronic fatigue syndrome: a hypothesis focusing on the autonomic nervous system. Clin Sci 96:117-125 